\title{
Governance of Decentralized Autonomous Organizations
}

\author{
Galia Kondova, Renato Barba \\ University of Applied Sciences and Arts Northwestern Switzerland, Basel, Switzerland
}

\begin{abstract}
This paper draws parallels between the OECD principle on disclosure and transparency and the decentralized autonomous organization (DAO) governance on a blockchain. It provides an overview of the DAO concept based on self-executing smart contracts on a blockchain. The blockchain keeps a record of data and tranasctions in a decentralized and immutable form. The self-executing smart contracts on a blockchain ensure transparency and automation of the decision-making process in a DAO. The paper concludes that a DAO governance structure provides for transparency and enables shareholders to exercise their rights in an informed way. Considering the early stage of development of DAOs, however, caution is needed especially with regards to potential protocol vulnerabilities and legal uncertainties.
\end{abstract}

Keywords: DAOs, blockchain, corporate governance, smart contracts

\section{Introduction}

Emerging technologies such as the blockchain promise innovation and enable new governance models that strongly build on trust and transparency. A new form of a blockchain-based organization, the decentralized autonomous organization (DAO), promises a fully democratic structure without hierarchies, which is completely controlled by decisions of all members of the organization. The decision-making process in DAOs takes place in a transparent way through self-executing smart contracts. This characteristic of DAOs is particularly conducive to the successful enforcement of one of the OECD Principles of Corporate Governance, namely the disclosure and transparency in the governance of any company (OECD, 2015).

This paper is the first one known to the authors to draw parallels between the OECD principle on disclosure and transparency and the DAO governance on a blockchain. It provides an overview of the DAO concept based on self-executing smart contracts on a blockchain. The blockchain keeps a record of data and tranasctions in a decentralized and immutable form. The self-executing smart contracts on a blockchain ensure transparency and automation of the decision-making process in a DAO. In particular, the paper argues that a DAO governance structure ensures real transparency and enables shareholders to exercise their rights in an informed way.

\section{Corporate Governance and Blockchain}

Corporate governance defines a legal and regulatory framework for the management and supervision of companies aimed at the sustainable interests of the company and its stakeholders. While maintaining the

Galia Kondova, Ph.D., Senior Lecturer, School of Business, University of Applied Sciences and Arts Northwestern Switzerland, Basel, Switzerland.

Renato Barba, M.Sc., Business Information Systems, School of Business, University of Applied Sciences and Arts Northwestern Switzerland, Basel, Switzerland. 
decision-making ability and efficiency at the highest corporate level, a well-governed company strives for transparency and a balanced relationship with all stakeholders (Vannod, Vannod, Druck, Daz, \& Albisrieden, 2002).

Good corporate governance is considered to be built on several guiding principles addressing areas like the rights and equitable treatment of shareholders; institutional investors, stock markets, and other intermediaries; the role of stakeholders; disclosure and transparency; and responsibilities of the board (OECD, 2015). In particular the OECD principle on disclosure and transparency stresses out that "a strong disclosure regime that promotes real transparency is a pivotal feature of market-based monitoring of companies and is central to shareholders' ability to exercise their shareholder rights on an informed basis” (OECD, 2015, p. 37).

At the same the major characteristics of blockchain like immutability, integrity, decentralization, and transparency (Zbinden \& Kondova, 2018) seem to provide a conducive basis for a successful enforcement especially of the OECD principle on disclosure and transparency. In particular "for shareholders, blockchains could offer lower costs of trading and more transparent ownership records, while permitting visible real-time observation of transfers of shares from one owner to another” (Yermack, 2017, p. 9 ).

\section{Decentralized Autonomous Organizations (DAOs)}

\section{Decision Making Process in DAOs}

DAOs are decentralized entities such as corporations and institutions running entirely autonomously and decentralized on a blockchain. Important management decisions are made by logic defined in code, executed by smart contracts and finally, through automation of decision-making, creating value for customers (Kypriotaki, Zamani, \& Giaglis, 2015). Furthermore, in DAOs there could be repetitive and simple tasks, which could be automated, and tasks, which need creative thinking, innovation, and responsibility. These second type of tasks require human engagements, which are incentivized by the code as well (DuPont, 2017).

According to BlockchainHub (2018), organizations need to fulfill the following requirements to identify as a DAO:

- Transaction tokens-a DAO should contain a valuable property in form of tokens, which could be used for rewards and that could represent the voting power of each member.

- Autonomous execution - the DAO as an entity acts independently and cannot be influenced by external forces. The organization and its open-source code are fully transparent and therefore incorruptible. The functionalities and program rules are written in code and maintained on the blockchain.

- Consensus - stakeholders need to fully agree if decisions need to be made, for example if a member wants to withdraw funds. Furthermore, bugs and optimizations need a democratic voting and decision-making, which can be seen as security issues if bugs are corrected too late.

- Contractors-in order to accomplish tasks and reach business goals, the DAO "hires" manufacturerers, developers, and other contractors based on shareholders' decisions as well.

- Proposals - the decision-making process always starts with a proposal. A proposal could also require monetary deposit to prevent a network overload of proposals.

- Voting - this step is crucial, as activities like money raising, investing, and repairing can only take place if a majority vote is reached.

The voting process in DAOs provides also an alternative to the current state of hierarchic and managerial decision-making in traditional companies. A DAO could have various stakeholders and each would have voting 
rights on how to allocate the organization's funds. Funds could be used for paying salaries or as internal currency to reward work. Voting rights could be distributed either based on the amount of tokens owned or evenly.

\section{DAOs and Smart Contracts}

From a technical point of view, a DAO represents one or more smart contracts, which are executed on top of a distributed consensus technology such as the Ethereum blockchain. Ethereum provides a blockchain with a built-in turing-complete programming language, where everyone can build decentralized applications based on own rules of ownership, transaction formats, and state transition functions, that execute when certain conditions are met. The transaction fees for smart contracts are paid in Ethereum's token Ether (Buterin, 2013).

Messages on Ethereum can either be transactions executed from external entities or internal contracts and can contain data for the recipient. An externally sent message is a transaction, containing the recipient of the message, the sender's signature, the amount that is sent and two values, Startgas and Gasprice. Gasprice is the fee paid for the miner per computational step for the transaction execution and Startgas is set to limit the computational steps, which prevents exponential blowup and infinite loops in code. If the transaction runs out of gas, all changes get reverted (Buterin, 2013). The state changes are illustrated in Figure 1. The transaction contains the sender's and recipient's address, the value sent in Ether, the additional sent data, and the signature.

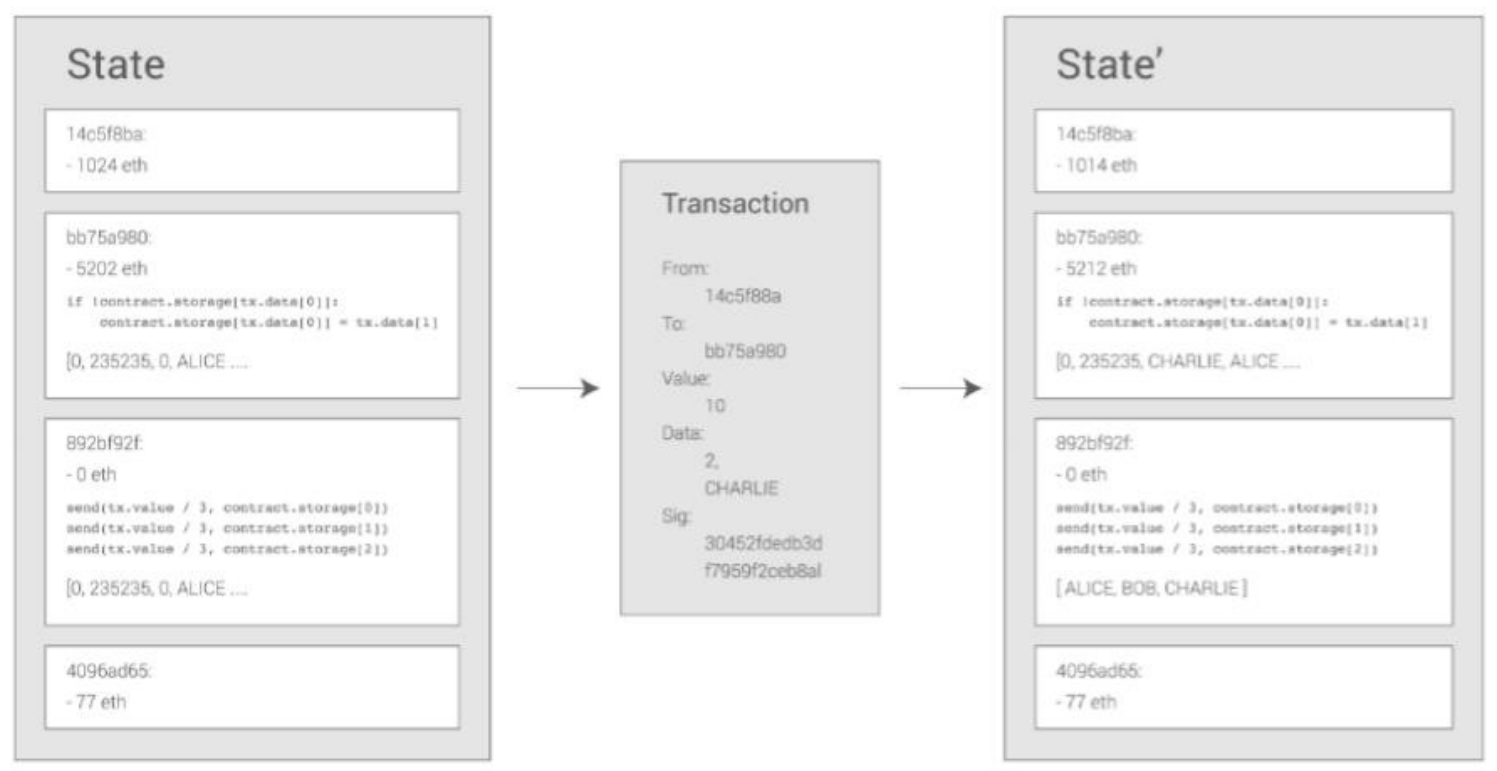

Figure 1. Ethereum transaction (Buterin, 2013).

What makes the concept of smart contracts appealing is that contracts have the same power as external accounts, including the creation of other contracts and sending messages autonomously. When orchestrating several smart contracts within a network, a complex system of functionalities is built, which executes autonomously. Furthermore, the Ethereum blockchain provides the underlying backend technology for recording transactions and logics in finding consensus. The layers are illustrated in Figure 2. 


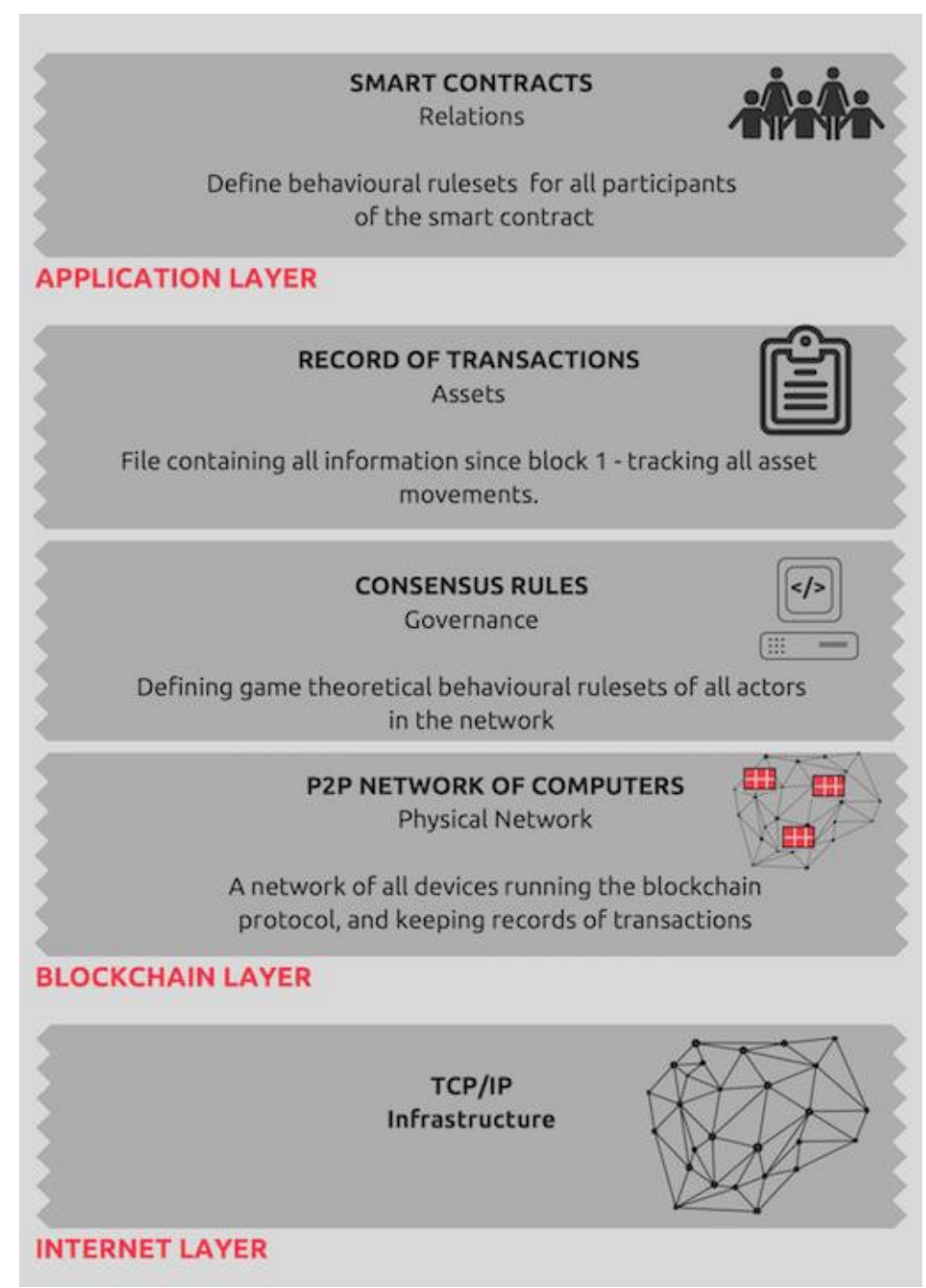

Figure 2. The architectural layers for smart contracts (BlockchainHub, 2018).

Before DAOs can establish themselves as companies, however, there are still some legal challenges ahead. First, DAO has no national location as it can operate decentralized and worldwide. This already raises questions about what form of society a DAO would represent and what laws a DAO and its shareholders would need to obey (Kypriotaki et al., 2015).

\section{Protocol Vulnerabilities: The DAO Attack}

The first DAO did not have a promising start. The protocol security problems resulted in shutting down the DAO.

In 2016, “The DAO” project was launched to enable investors to directly finance and manage new companies on the Ethereum blockchain. The investment share of the participants had a direct correlation with the number of votes in organizational decisions. At first it seemed like an optimal system, without inefficient human negotiations where all regulations were defined by program code (DuPont, 2017).

With the equivalent of 250 million dollars in Ether (ETH), the DAO went live and thus broke all previous crowdfunding records. Shortly after the go-live, the DAO was hacked by exploiting an unintentional behavior 
of the code's logic by an unknown person. Millions of dollars in ETH were drained and redirected to a different Ethereum account. After the intervention of the Ethereum Foundation, the money was refunded by performing a hard fork and returning the money to its original owners (DuPont, 2017).

\section{Conclusion}

The corporate governance principles provide guidance for managing the company in the long-term interest of the stakeholders and in a transparent manner. In particular, "the corporate governance framework should ensure that timely and accurate disclosure is made on all material matters regarding the corporation” (OECD, 2015, p. 37).

At the same time, the DAO concept of corporations and institutions running entirely autonomously and decentralized on a blockchain through self-executing smart contracts seems to provide the necessary framework for a successful enforcement of the transparent governance principle.

However, considering the early stage of development of DAOs, caution is needed especially with regards to potential protocol vulnerabilities and legal uncertainties related to DAOs.

\section{References}

BlockchainHub. (2018). What is a drom https://medium.com/universablockchain/decentralized-autonomous-organization-what-is-a-dao-company-eb99e472f23e

Buterin, V. (2013). A next generation smart contract and decentralized application platform. Retrieved from https://cryptorating.eu/whitepapers/Ethereum/Ethereum_white_paper.pdf

DuPont, Q. (2017). Experiments in algorithmic governance: A history and ethnography of “The DAO”, a failed decentralized autonomous organization. In Bitcoin and beyond: Cryptocurrencies, blockchains, and global governance (pp. 157-177). Retrieved from https://doi.org/10.4324/9781315211909

Kypriotaki, K., Zamani, E., \& Giaglis, G. (2015). From bitcoin to decentralized autonomous corporations-extending the application scope of decentralized peer-to-peer networks and blockchains. Retrieved from https://doi.org/10.5220/0005378402840290

Nakamoto, S. (2009). Bitcoin: A peer-to-peer electronic cash system. Retrieved from www.bitcoin.org

OECD. (2015). G20/OECD principles of corporate governance. Retrieved from https://doi.org/10.1787/9789264236882-en

Vannod, A., Druck, S. G., Daz, \& Albisrieden, D. (2002). Swiss code of best practice for corporate governance. Retrieved from https://www.economiesuisse.ch/sites/default/files/publications/economiesuisse_swi sscode_d_web.pdf

Yermack, D. (2017). Corporate governance and blockchains. Review of Finance, 21(1), 7-31. Retrieved from https://doi.org/10.1093/rof/rfw074

Zbinden, F., \& Kondova, G. (2019). Economic development in Mexico and the role of blockchain. Advances in Economics and Business, 7(1), 55-64. doi:10.13189/aeb.2019.070106 